This PDF is a selection from an out-of-print volume from the National Bureau of Economic Research

Volume Title: Statistical Indicators of Cyclical Revivals and Recessions

Volume Author/Editor: Geoffrey H. Moore

Volume Publisher: NBER

Volume ISBN: 0-87014-346-8

Volume URL: http://www.nber.org/books/moor50-1

Publication Date: 1950

Chapter Title: Criteria for Selecting Indicators

Chapter Author: Geoffrey H. Moore

Chapter URL: http://www.nber.org/chapters/c3231

Chapter pages in book: (p. 20 - 31) 
Thus even the series in this sample, selected for the regularity with which they conform to business cycles, show wide differences in the timing of their cyclical fluctuations. The percentage of series expanding rarely exceeded 80 or fell below 20 in any month between 1885 and 1940 . The zones in which the peaks (or troughs) concentrate extend over a year or two or three, and the peak zones often overlap the trough zones. In the words of Mitchell and Burns (Bulletin 69, p. 2), a business cycle revival or recession "is not an event that happens in a single month, but a complicated series of changes that occur cumulatively in various economic processes during a period that may last a year or more". This very fact spells some hope for the user of statistical indicators.

\section{Criteria for Selecting Indicators}

Bulletin 69 described an ideal statistical indicator of cyclical revivals and recessions in the following terms:

1) It would cover half a century or longer, thus showing its relation to business cycles under a variety of conditions.

2) It would lead the month around which cyclical revival centers by an invariable interval-say three months, or better, six months. It would also lead the central month of every cyclical recession by an invariable interval, which might differ from the lead at revival.

3) It would show no erratic movements; that is, it would sweep smoothly up from each cyclical trough to the next cyclical peak and then sweep smoothly down to the next trough, so that every change in its direction would herald the coming of a revival or recession in general business.

4) Its cyclical movements would be pronounced enough to be readily recognized, and give some indication of the relative amplitude of the coming change.

5) It would be so related to general business activity as to establish as much confidence as the nature of such things allows that its future behavior in regard to business cycles will be like its past behavior.

As is, of course, obvious from Table 2 and Chart 1, no series possessing all these characteristics was found. Nor have we had 
better success in this investigation. The synthetic curve plotted in Chart 3 (the percentage of series expanding) comes perhaps as close as any to meeting the specifications, but even it does not lead the central months of cyclical revivals and recessions by an invariable interval nor is it free from erratic movements. Its merits will be discussed more fully in Section 6 .

Realizing that the ideal indicator was not to be found in their collection, the authors of Bulletin 69 looked for series that most nearly approached the ideal. The criterion for their initial selection of 71 indicators was what may be called the 'two-thirds rule'. That is, a series was considered an acceptable indicator of revivals if its specific cycle troughs led the corresponding reference troughs at two-thirds or more of the reference troughs it covered; or if it was 'roughly coincident' (turned within three months of the reference trough) at two-thirds or more of the troughs; or even if it lagged at two-thirds or more of the troughs. ${ }^{9}$ Of course, if an ideal leading indicator could be found, the other types would be superfluous. As it is, both the roughly coincident and lagging series yield useful information on the course of a business cycle.

For the further screening given the 71 indicators selected by the two-thirds rule (which was relaxed slightly in a few cases), the five general criteria quoted above were spelled out into eleven specific characteristics. Other things being equal, a series was considered more useful as an early indicator of revivals

1) The longer its average lead at past revivals,

2) The more uniform these leads are in occurrence and length,

3) The closer its specific cycles come to having a one to one correspondence with business cycles,

4) The more clearly defined its specific cycles,

5) The less intense its erratic movements in comparison with the amplitude of its specific cycles,

9 The peaks of a series taken to be inverted with respect to business cycles, such as business failures, are compared with reference troughs. Usually it is easy to decide whether a series should be treated positively or invertedly; but it is difficult when the series leads or lags by long intervals. Throughout this report we adhere to the objective rule we follow in our standard analyses (cf. Measuring Business Cycles, pp. 115-7, 188-9). For example, the series on bond sales (Chart 2) is treated invertedly under our rule; hence most of the timing observations are lags: If it were treated positively it would lead. Adherence to the rule does not, of course, necessarily lead to the most useful or sensible treatment. 
6) The fewer the changes in the direction of its month to month movements,

7) The smaller and more regular the seasonal variations that have to be 'eliminated' before the specific cycles can be studied,

8) The larger the number of revivals covered,

9) The further back in time any irregularities in conformity to business cycle revivals occurred,

10) The broader the range of activities represented,

11) The more stable the economic significance of the process represented.

A sifting of the 71 series on the basis of these criteria gave the 21 listed in Table 2.

Clearly, many considerations enter into the selection of a series as an indicator of revival or recession. Some that have nothing to do with the behavior of the series might be added to the eleven noted. For example, a lag in the publication of a series is often sufficient to justify its rejection as a useful indicator. Again, whether or not the series is published in seasonally adjusted form is a consideration, for seasonal elimination is expensive and timeconsuming.

In this study we have not yet carried the application of selective criteria as far as in Bulletin 69, though we plan to continue work along these lines. What we have done is to design and apply a method for rating series on the basis of two criteria: the consistency with which their movements have conformed to business cycles, and the consistency with which their turning points have led, lagged, or roughly coincided with the reference dates. A series' rating is based upon estimates of the probability that, for specified measures of its past performance, a result as good as or better than the one observed would occur in random sampling.

These measures of performance may be based on all the cycles covered by a series; then the computed probability takes into account differences in the lengths of series, i.e., differences in the amount of information on cyclical behavior available for each series. The two-thirds rule did not do this. On the other hand, to avoid ambiguities due to differences in time coverage and to possible changes in behavior, it is desirable to have measurements based upon a fixed and recent period for all series. Consequently, two sets of measures have been used: one based on all cycles cov- 
ered by the series, the other on 1919-38, a period covered by most of the series.

The first criterion, conformity, is designed to spot series whose movements have paralleled business cycles consistently. If a series consistently expands during business expansions and contracts during business contractions, it has at least one of the qualities of a good indicator. However, allowance should be made for the fact that some series typically contract during business expansions and expand during contractions, and that others show characteristic differences in timing in relation to business cycles.

These differences are revealed, at least crudely, by the reference cycle patterns computed for each series (cf. Chart 2). Consequently, we examine the patterns to determine the set of 'stages', or interval, during which the series typically expands, and those during which it typically contracts. ${ }^{10}$ For example, the expansion intervals for the first six series in Chart 2, taking into consideration all the cycles covered by each series, are: residential building contracts, VII-IV; industrial production index, I-V; locomotive shipments, III-VI; liabilities of business failures, IVVII; refined copper stocks, V-IX; bond sales, VII-II. The seventh series in Chart 2, agricultural marketings, behaves so irregularly in successive reference cycles that a division into typical expansion and contraction intervals is not justified.

The expansion interval usually corresponds rather closely to the interval during which the average reference pattern rises, and the contraction interval to the interval during which the average pattern declines, as the reader can verify from Chart 2 . The intervals, of course, imply a certain kind of timing. Thus the expansion interval, VII-IV, for residential building contracts implies that the series typically leads by one stage at business peaks and by two stages at business troughs. The typical sequences among the series can be traced by the arrangement that follows, which utilizes the expansion and contraction intervals.

Once the expansion and contraction intervals have been fixed, one can measure the consistency of performance in successive cycles by counting the number of cycles in which the series rises between the initial and terminal expansion stages and the number in which it falls; likewise one can count the number of cycles in which there is a decline and the number in which there is a rise between the initial and terminal contraction stages.

10 For an account of how this is done the reader is referred to Measuring Business Cycles, pp. 185-97. 
Resid. bldg. contracts

Indus. prod. index

Locomotive ship.

Failures, liab., mfg.

Ref. copper stocks

Bond sales, N. Y. S. E.
TYPICAL DIRECTION OF CHANGE BETWEEN STAGES

I-II II-III III-IV IV-V V-VI VI-VII VII-VIII VIII-IX

BUSINESS EXPANSION BUSINESS CONTRACTION

Trough First Middle Last Peak First Middle Last

$\begin{array}{cccc}\text { to to } & \text { to third to to to third } \\ \text { first middle last to first middle last to }\end{array}$

third third third peak third third third trough

$\begin{array}{llllllll}+ & + & + & - & - & - & + & + \\ + & + & + & + & - & - & - & - \\ - & - & + & + & + & - & - & - \\ - & - & - & + & + & + & - & - \\ - & - & - & - & + & + & + & + \\ + & - & - & - & - & - & + & +\end{array}$

In Chart 2 the industrial production index has a perfect conformity score: it rises during its expansion interval in each of the five cycles (that is, the standing at stage $\mathrm{V}$ is higher than at stage $\mathrm{I}$ in five cycles); and it declines between $\mathrm{V}$ and IX in each cycle. Refined copper stocks rise in four out of five cycles during their expansion interval, V-IX, and decline in four cycles out of five during their contraction interval. Bond sales rise three times out of five during their expansion interval, VII-II,${ }^{11}$ and decline four times out of five during their contraction interval.

The expansion and contraction conformity scores, when combined, measure the parallelism between the movements of the series and business cycles. For this purpose, the number of rises in the series' typical expansion interval and declines in its contraction interval can be taken as a proportion of the total number of expansions and contractions covered. This measure would be $\frac{10}{10}$ or unity for the index of industrial production, $\frac{8}{10}$ for copper stocks, $\frac{7}{10}$ for bond sales. It could not exceed unity, nor could it fall much below one-half if the expansion interval was at all typical.

However, this simple measure is the same for a series lapsing once from perfectly consistent conformity in five phases as for a series lapsing twice out of ten. Should one not have more confidence in the excellence of the series in the second case than in the first? If some simplifying assumptions are made, it is easy to compute the probability that as few as, say, two lapses in conformity out of ten will occur by chance, and this may be compared with the

11 In this case, to compare stage VII of one cycle with stage II of the next, the standing at stage II is recomputed on the base of the preceding cycle. 
probability of obtaining as good a result as one lapse out of five by chance. Our method, then, is simply to compute these probabilities for each series' expansion and contraction scores, and to combine the expansion and contraction probabilities for a given series by multiplying them: the smallest product probabilities indicate the best series so far as conformity is concerned. ${ }^{12}$

A small product probability may, however, result from a strong secular trend in the series. An upward trend can help to produce a high expansion score, and the corresponding expansion probability may be so low as to offset a relatively high contraction probability and yield a relatively low product. Two procedures help to avoid this. First, we require that there be both an excess of rises over declines in expansion intervals and an excess of declines over rises in contraction intervals. This eliminates series in which the effect of trend is so strong as to produce more rises than declines in both phases (or vice versa). Such series cannot be said to parallel business cycles. Although they may reflect the influence of business cycles by alternately rising faster and slower, and hence not be entirely valueless as indicators, they may well be set aside at the start.

The second and more important procedure is to subject the expansion and contraction probabilities to a maximum acceptance level, .188. When either probability is above this level (or rather, above the nearest possible approximation to it in the given case), the series is rejected. The .188 level is the probability that one or fewer lapses in conformity will occur in five phases. Series that would get a low product probability merely because of an exceptionally low expansion (or contraction) probability are thereby eliminated. Reasonably good conformity to both ex-

12 The underlying assumptions are that the probability that a series will rise (or fall) during its expansion interval in a given business cycle is one-half, and that the results in successive cycles are independent. Then the probability that no lapses will occur in, say, 5 phases is $(1 / 2)^{5}=.03125$; that 1 lapse will occur, $5(1 / 2)^{5}=.15625 ; 2$ lapses, $10(1 / 2)^{5}=.3125$; and so on according to the binomial expansion. Cumulating, one obtains the probability for as good a result as no lapses, .03125 ; one lapse, .1875 ; two lapses, .5 ; etc. Both underlying assumptions are open to question, and the computed probabilities cannot be interpreted in any strict sense. Several other procedures were tried but the above seemed adequate for rating series roughly, which is all that is possible since the conformity scores themselves contain a rather limited amount of information about the series' behavior. When a series does not change in its expansion (or contraction) interval in a given cycle the observation is counted as one-half rise, one-half fall. 
pansions and contractions is an essential characteristic of a good indicator, whether revivals or recessions are of chief interest.

The general effect of the procedure, as the accompanying figures illustrate, is that for the same proportion of lapses in conformity the longer the series the lower the probability; and the longer the series the higher the maximum acceptable proportion of lapses in conformity. When the conformity probability cri-

Phases covered by series, no.

$\begin{array}{rrrr}5 & 10 & 15 & 20 \\ 1 & 2 & 3 & 4 \\ .188 & .055 & .018 & .0058 \\ 1 & 3 & 51 / 2 & 71 / 2 \\ .188 & .172 & .214 & .182\end{array}$

Lapses in conformity, no.

Probability

Maximum acceptable no. of lapses

Probability

terion is applied to the full periods covered by the seven series of Chart 2, two series are rejected (Table 3 ).

Series with acceptable conformity are then subjected to the timing criterion. Timing is measured by comparing the dates of a series' specific cycle peaks and troughs with the reference dates, and the intervals, in months, are classified as leads, lags, or rough coincidences. ${ }^{13} \mathrm{~A}$ consistent leader at peaks, then, is a series that leads at a large proportion of the reference peaks covered. What is a large proportion and what a small is determined by computing the probability that, when a series covers a certain number of reference peaks (or troughs), a specified number of timing comparisons of a given type will be equaled or exceeded by chance. ${ }^{14}$ As with conformity, a maximum acceptance level is adopted, corresponding as nearly as may be to the probability $(.223)$ that four or more timing comparisons will appear in a given group when the series covers six reference turns. The accompanying figures illustrate how the timing probabilities vary with the length of the

${ }^{18}$ For purposes of the probability criterion described below, an exact coincidence was counted as a half lead and a half lag. Rough coincidences include exact coincidences and leads or lags of one, two, or three months. Any timing comparison must be in one of the three timing groups, and some may be in two (leads and rough coincidences, or lags and rough coincidences). But at some reference turns there may not be any timing comparison, either because no specific cycle turn occurs in the vicinity or because several turns compete. Cf. Measuring Business Cycles, pp. 116-28.

14 Since a tabulation of the total number of leads, lags, and rough coincidences for all series having acceptable conformity revealed that the number in each class was roughly the same-about 43 percent of the number of reference turns covered by the series, .43 was adopted as the probability that a series will produce a timing comparison of a given type at a reference turn. 


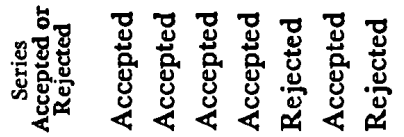

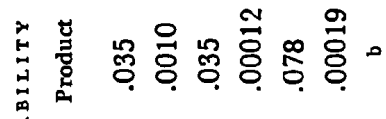

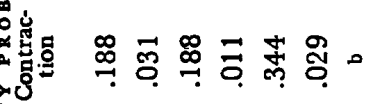

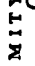

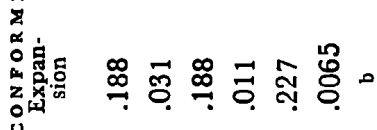

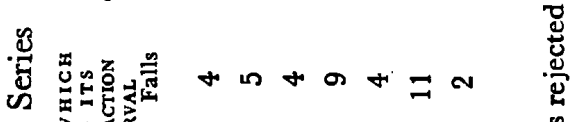

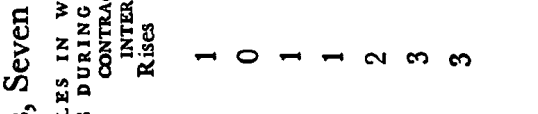

$\infty$

象

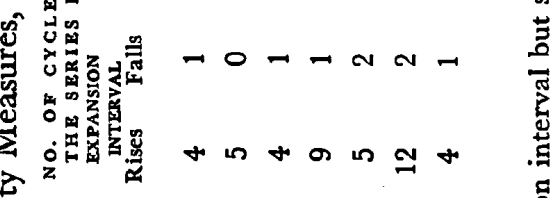

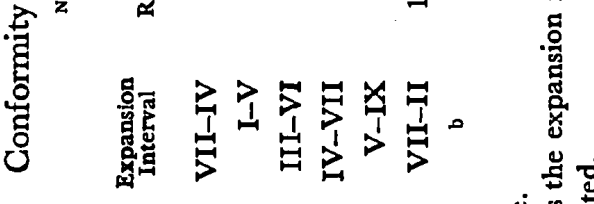

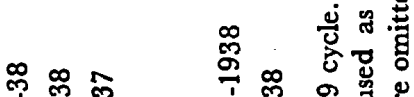

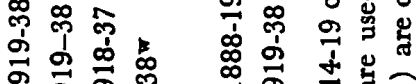

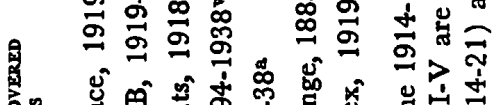

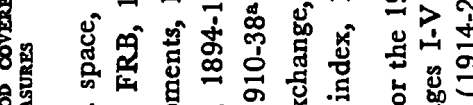

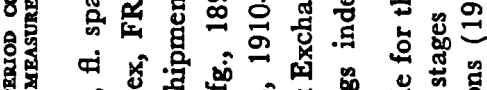

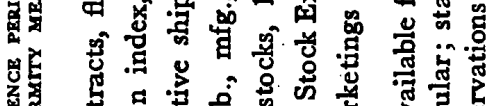

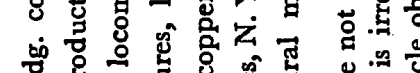

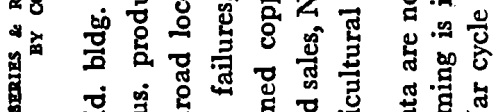

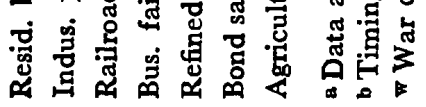


series. A series leading at two-thirds of the reference peaks covered is assigned a much lower probability if it achieves this record in 21 cycles than in 6 . A record of leads at 11 peaks in 21 cycles

No. of ref. peaks (or troughs) covered by series

Timing comparisons of one type, no. Probability

$\begin{array}{rrrr}6 & 12 & 18 & 21 \\ 4 & 8 & 12 & 14 \\ .223 & .087 & .037 & .025 \\ & & & \\ 4 & 7 & 10 & 11 \\ .223 & .216 & .210 & .257\end{array}$

Timing comparisons of one type, min. accept. no.

Probability

is roughly equivalent, on a probability basis, to a record of 4 in 6 cycles. ${ }^{15}$ Table 4 shows the timing probabilities for the five series of Chart 2 that have acceptable conformity.

Since the conformity and timing probabilities just described are based on a series' behavior during all the business cycles it covers, ${ }^{16}$ they do not take account of possible secular changes in cyclical behavior. For example, a series whose conformity has improved may get a lower rating than its recent performance would justify. Or a series may be classed as a leader at peaks, when recently it has lagged. While significant shifts of this sort do not appear to be widespread, our measures may be misleading when they do occur.

A few illustrations will suffice to reveal the nature of the problem. A series on railroad freight ton-miles is rejected on the basis of its conformity during all cycles covered (1867-1938), because in nearly half the reference contraction intervals it does not decline. But its failure is confined to its early years; since 1907 it has declined in every contraction except one, that of 1910-12, a rather mild depression. This shift in behavior is due to the de${ }_{15} \mathrm{~A}$ series that leads at 11 peaks out of 21 has not, it is true, achieved a good record as a leader if it lags at the other 10. Our method, in effect, gives some weight to the possibility that the series will not reach a corresponding peak at all 21 reference peaks. The difficulty could be avoided by computing two probabilities, one based on the number of corresponding turns relative to the number of reference turns, the other on the number of leads relative to the number of corresponding turns.

A simpler expedient would be to adopt a higher acceptance level. The probability (.057) corresponding to 5 leads out of 6 reference turns covered would be fairly satisfactory, since it would require a minimum of 13 leads in 21 turns $(P=.064)$. The classification of series in Sec. 5 and 6 is based, however, on the procedure described in the text. A saving feature is that a well conforming series with, say, 11 leads and 10 lags is likely to have at least as many rough coincidences, in which case it will be classified also as a 'coincider'.

${ }^{16}$ An exception is made in price and certain value series, observations during war cycles being omitted. 


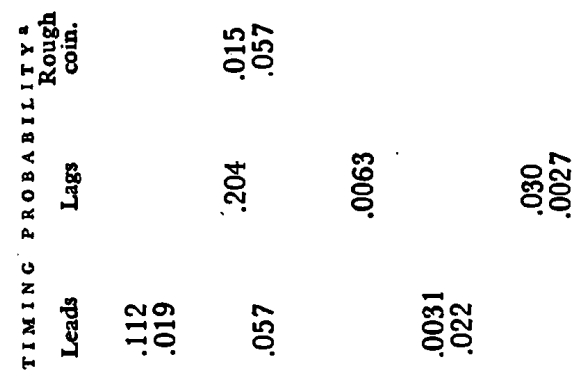

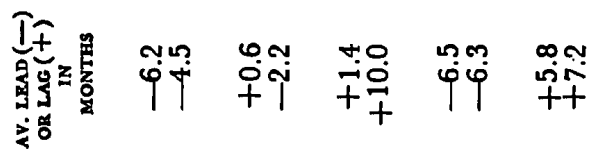

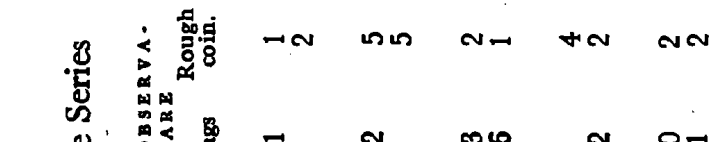
. 井导 \&

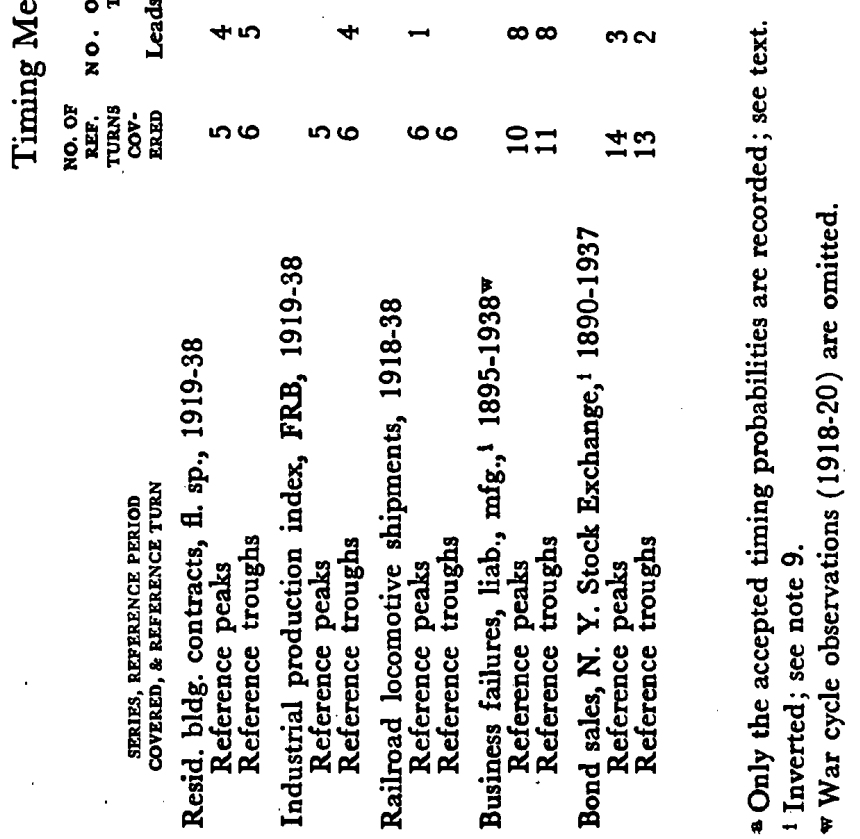


clining secular rate of growth of railroad freight traffic (the rate of growth in the early years being so rapid as to offset depressions) and perhaps also, in some degree, to the declining relative volume of agricultural traffic.

Shifts in conformity are often related to shifts in timing. An improved conformity may mean a more consistent timing relationship, i.e., the proportion of reference turns for which there are no corresponding specific turns may be smaller, and noncorresponding, or extra, specific turns may be less frequent. For example, in 1867-1919 total exports conformed irregularly, rising in 10 and falling in 3 expansions and rising in 9 and falling in 4 contractions; since 1919 exports have increased in each expansion and declined in each contraction. The pre-1919 period yields only 11 timing comparisons; 15 reference turns are skipped and there are 24 extra specific turns. After 1919 there are 7 comparisons; only 4 turns are skipped and there are only 5 'extras'.

Besides shifts in the degree of correspondence between specific and business cycles, shifts may occur in the kind of timing. Prior to 1913 railroad ton-miles lagged at each of the 8 reference peaks with which a specific peak could be matched, often by substantial intervals. Since then it has led at 4 peaks, and lagged only twice; none of these intervals exceeded four months. Orders for railway equipment illustrate another type of shift in timing. In the early years they led business revivals by much longer intervals than in recent cycles. Burns and Mitchell (Measuring Business Cycles, pp. 414-6) attribute this to a shift of the railroad industry from a dominant to a subordinate position as an object of investment.

The method we have chosen to meet the problem of shifts in conformity and timing is to rate all series on the basis of their performance after 1919, provided they cover at least the four cycles 1921-38. Comparison with results based on all cycles covered will bring to light changes in behavior. Furthermore, by rating all series on the basis of performance in 1919-38 we can test the performance of identical groups of series in earlier cycles; in other words, test whether secular or other changes in behavior have been so general as to make it impossible to discover stable behavior characteristics. Looking backward in time, we can make the same sort of test that was made in Table 2 looking forward. Another advantage of fixing the period (approximately) is that the arbitrary method of equating different historical periods by a probability scheme is avoided. In particular, since the dates of 
reference troughs seem to have a small bias in the early years, long series are in fact not treated on precisely the same basis as short, in respect of their behavior at troughs. The reference dates in 1919-38 are more firmly established and in any event fixing the period eliminates the difficulty.

The conformity and timing probabilities (for the full period covered by the series and for 1919-38) are the measures so far developed and applied in this study. Both types of measure contribute useful information about the quality of a series as an indicator. While they are not strictly independent in a statistical sense, they utilize the data in quite different ways, and the two together contribute more information than either alone. Nevertheless, by themselves they will not yield a wholly satisfactory set of indicators. For one thing, the acceptance levels we have set are met by very many series, and a long list of indicators is practically a serious inconvenience. But we did not wish to set the levels much higher (see, however, note 15), for that would mean giving disproportionate weight to what are, in and of themselves, rather rough measures of behavior. We plan, instead, to apply additional criteria to achieve a finer selection. Some consideration, for example, should be given to the length of the average lead or lag; this is done only indirectly and crudely by the timing probabilities (see Sec. 5). Again, a series may get a low conformity and a low timing probability, yet have such large erratic movements as to be of little value as an indicator. Moreover, account has not been taken directly of how closely the movements of a series match the variations in amplitude of successive business cycles. Consequently, the classifications of series based upon the probability measures, which we present and utilize in Sections 5 and 6 , must be taken as provisional and subject to elaboration and revision.

\section{Classification of Series According to Conformity And Timing}

The 801 monthly and quarterly time series for the United States analyzed in connection with the National Bureau's general investigation of business cycles are the foundation of this study. ${ }^{17}$ Assembled over a period of years for a variety of purposes, they ${ }^{17}$ Included are 57 series on the status of national banks at 5 irregularly spaced 'call dates' within the year; 61 'short' series are excluded (see text). 